\title{
SHORTED OPERATORS RELATIVE TO A PARTIAL ORDER IN A REGULAR RING
}

\author{
BRIAN BLACKWOOD, S. K. JAIN, K. M. PRASAD, AND ASHISH K. SRIVASTAVA
}

\begin{abstract}
In this paper, the explicit form of maximal elements, known as shorted operators, in a subring of a von Neumann regular ring has been obtained. As an application of the main theorem, the unique shorted operator (of electrical circuits) which was introduced by Anderson-Trapp has been derived.
\end{abstract}

\section{INTRODUCTION}

Various partial orders on an abstract ring or on the ring of matrices over the real and complex numbers have been introduced by several authors either as an abstract study of questions in algebra, or for the study of problems in engineering and statistics (See, e.g. 11, 2], 4, 7], 12, and [13). Also, a partial order on semigroups is studied by several authors (See, e.g. [6], [15], and [16]). In this paper we study the well-known minus partial order on a von Neumann regular ring which is simply a generalization of a partial order on the set of idempotents in a ring introduced by Kaplansky. For any two elements $a, b$ in a von Neumann regular ring $R$, we say $a \leq^{-} b$ (and read it as $a$ is less than or equal to $b$ under the minus partial order) if there exists an $x \in R$ such that $a x=b x$ and $x a=x b$ where $a x a=a . \quad$ Furthermore, we define the partial order $\leq^{\oplus}$ by saying that $a \leq^{\oplus} b$ if $b R=a R \oplus(b-a) R$, and call it the direct sum partial order. The Loewner partial order on the set of positive semidefinite matrices $S$ is defined by saying that for $a, b \in S, a \leq_{L} b$ if $b-a \in S$. The direct sum partial order is shown to be equivalent to the minus partial order on a von Neumann regular ring. It is known that the minus partial order on the subset of positive semidefinite matrices in the matrix ring over the field of complex numbers implies the Loewner partial order. The main result of this paper gives an explicit description of maximal elements in a subring under minus partial order (Theorem 13). As a special case, we obtain a result similar to the one obtained by Mitra-Puri (13), Theorem 2.1) for the unique shorted operator; which, in turn, is equivalent to the formula of Anderson-Trapp ([2, Theorem 1) for computing the shorted operator of a shorted electrical circuit (Theorem 17).

\section{Definitions}

Throughout this paper, $R$ is a ring with identity. An element $a \in R$ is called von Neumann regular if $a x a=a$ for some $x \in R$ and $x$ is called a von Neumann inverse of $a$. We will denote an arbitrary von Neumann inverse of $a$ by $a^{(1)}$. An element

2000 Mathematics Subject Classification. 06A06, 06A11, 15A09, 16 U99.

Key words and phrases. von Neumann regular ring, partial order, shorted operator. 
$a \in R$ is called weakly regular if $x a x=x$ for some $x \in R$ and $x$ is called a weak von Neumann inverse of $a$. We will denote a weak von Neumann inverse of $a$ by $a^{(2)}$. If $a x a=a$ and $x a x=x$, then $x$ is called a strong von Neumann inverse of $a$. We will denote a strong von Neumann inverse of $a$ by $a^{(1,2)}$. A ring $R$ is called von Neumann regular if every element in $R$ is von Neumann regular. For convenience, we will use the terminology regular ring in place of von Neumann regular ring. For details on regular ring, the reader is referred to $[5]$.

Let $S$ be the set of all regular elements in any ring $R$. For $a, b \in S$ we say that $a \leq^{-} b$ if there exists a von Neumann inverse $x$ of $a$ such that $a x=b x$ and $x a=x b$. This is known as the minus partial order as stated above for regular rings. The minus partial order clearly generalizes the definition of Kaplansky according to which if $e, f$ are idempotents then $e \leq f$ if $e f=e=f e$.

We remark that for the ring of matrices over a field, it is known that $a \leq^{-} b$ if and only if $\operatorname{rank}(b-a)=\operatorname{rank}(b)-\operatorname{rank}(a)$.

Let $T$ be a ring with involution *. If $x$ is a strong von Neumann inverse of $a$ such that $(a x)^{*}=a x,(x a)^{*}=x a$ and $a x=x a$ then $x$ is called the Moore-Penrose inverse of $a$ and is denoted by $a^{\dagger}$. Let $M$ be the set of positive semidefinite matrices. For $w \in M$ and $b \in T, x$ is called the unique $w$-weighted Moore-Penrose inverse of $b$ if $x$ is a strong von Neumann inverse of $b$ and satisfies $(w b x)^{*}=w b x$ and $(w x b)^{*}=w x b$. For details on Moore-Penrose inverse, one may refer to Rao-Mitra [17] or Ben-Israel and Greville [3].

\section{Preliminary Results}

The following result of Jain and Prasad ([8], Theorem 1) will prove to be useful throughout this paper and, specifically, for providing an equivalent definition of the minus partial order on a regular ring.

Theorem 1. Let $R$ be a ring and let $a, b \in R$ such that $a+b$ is a regular element. Then the following are equivalent:

(1) $a R \oplus b R=(a+b) R$;

(2) $R a \oplus R b=R(a+b)$;

(3) $a R \cap b R=(0)=R a \cap R b$.

From Rao-Mitra ([17, Theorem 2.4.1, page 26), we have the following nice characterization of $\left\{a^{(1)}\right\}$ and $\left\{a^{(1,2)}\right\}$.

Lemma 2. Let $R$ be a ring and let $a \in R$. If $x \in\left\{a^{(1)}\right\}$ then $\left\{a^{(1)}\right\}=x+(1-$ $x a) R+R(1-a x)$. In addition, $\left\{a^{(1,2)}\right\}=\left\{a^{(1)} a a^{(1)}\right\}$.

We now investigate properties of the direct sum partial order and its relation to the minus partial order.

Let $R$ be a regular ring. Recall $a \leq^{\oplus} b$ if and only if $b R=a R \oplus(b-a) R$. By Theorem 1, this is equivalent to $R b=R a \oplus R(b-a)$. It is straightforward to see that $\leq^{\oplus}$ is a partial order.

Next we show that the minus partial order is equivalent to the direct sum partial order on a regular ring. Hartwig-Luh showed that, when $R$ is a regular ring, (2) is equivalent to (3) with the additional hypothesis that $a \in b R b$ (see [14], page 5).

Lemma 3. Let $R$ be a regular ring and $a, b \in R$. Then the following are equivalent: 
(1) $a \leq^{\oplus} b$;

(2) $a \leq^{-} b$

(3) $\left\{b^{(1)}\right\} \subseteq\left\{a^{(1)}\right\}$.

Proof. (1) $\Longrightarrow(2):$ As $a \leq^{\oplus} b, b R=a R \oplus(b-a) R$. It follows that $a R \subseteq b R$. Hence, $a \in b R$ and thus $a=b x$ for some $x \in R$. As $R$ is a regular ring, for any $g \in\left\{b^{(1)}\right\}, b g b=b$. Thus $b g a=b g(b x)=(b g b) x=b x=a$. Now aga $=$ $b g a-(b-a) g a=a-(b-a) g a$. Thus $a-a g a=(b-a) g a$. But $a R \cap(b-a) R=(0)$ and $a-a g a=(b-a) g a \in a R \cap(b-a) R$. Hence $a-a g a=0$ and $(b-a) g a=0$. Therefore $a g a=a=b g a$ and hence $\left\{b^{(1)}\right\} \subseteq\left\{a^{(1)}\right\}$. Indeed, this demonstrates that $(1) \Longrightarrow(3)$. Now choose $x=g a g$. Then $a x a=a(g a g) a=a g a=a$ and $x \in\left\{a^{(1)}\right\}$. Now $b x=(b g a) g=a g$ as $b g a=a$. Furthermore, $a x=a g a g=a g$ as $a g a=a$. Thus $a x=b x$. Now $b g(b-a)=b g b-b g a=(b-a)$ and $(b-a) g(b-a)=$ $b g(b-a)-a g(b-a)=(b-a)-a g(b-a)$. Hence $a g(b-a)=(b-a)-(b-a) g(b-a) \in$ $a R \cap(b-a) R=(0)$. Thus $(b-a)=(b-a) g(b-a)$ and $a g(b-a)=0$. It follows that $a g b=a g a=a$. Now $x b=(g a g) b=g(a g b)=g a$ and $x a=g a g a=g a$. Therefore $x b=x a$. Thus $a x=b x$ and $x a=x b$ for some $x \in\left\{a^{(1)}\right\}$ and it follows that $a \leq^{-} b$.

$(2) \Longrightarrow(3)$ : This is well-known. We prove it here for completeness. As $a \leq^{-} b$, there exists some $x \in\left\{a^{(1)}\right\}$ such that $a x=b x$ and $x a=x b$. It follows that $a=a x a=b x a=a x b$ and for any $y \in\left\{b^{(1)}\right\}$, ay $a=(a x b) y(b x a)=a x(b y b) x a=$ $a x b x a=(a x b) x a=a x a=a$. Thus $\left\{b^{(1)}\right\} \subseteq\left\{a^{(1)}\right\}$.

(3) $\Longrightarrow(1)$ : Given that $\left\{b^{(1)}\right\} \subseteq\left\{a^{(1)}\right\}, a b^{(1)} a=a$ for any $b^{(1)} \in\left\{b^{(1)}\right\}$. By Lemma 2, $\left\{b^{(1)}\right\}=g+(1-g b) R+R(1-b g)$ for $g \in\left\{b^{(1)}\right\}$. For each $x \in\left\{b^{(1)}\right\}$ there exists some $r_{1}, r_{2} \in R$ such that $x=g+(1-g b) r_{1}+r_{2}(1-b g)$. Multiplying on the left and right by $a$ yields $a x a=a\left[g+(1-g b) r_{1}+r_{2}(1-b g)\right] a$. Hence $a=a x a=a\left[g+(1-g b) r_{1}+r_{2}(1-b g)\right] a=a g a+a(1-g b) r_{1} a+a r_{2}(1-b g) a=$ $a+a(1-g b) r_{1} a+a r_{2}(1-b g) a$. Thus $a(1-g b) r_{1} a+a r_{2}(1-b g) a=0$. As $a(1-g b) r_{1} a+a r_{2}(1-b g) a=0$ holds for all $r_{1}$ and $r_{2}$, we can take, in particular, $r_{2}=0$ which gives $a(1-g b) r_{1} a=0$ for all $r_{1}$ and hence $a(1-g b) R a=(0)$. Similarly, by taking $r_{1}=0$, we conclude $a R(1-b g) a=(0)$. Now $(a(1-g b) R)^{2}=$ $(a(1-g b) R)(a(1-g b) R)=(a(1-g b) R a)((1-g b) R)=(0)((1-g b) R)=(0)$. Similarly $(R(1-b g) a)^{2}=(0)$. Since $R$ is a regular ring, it has no nonzero nilpotent left or right ideal. Thus, $a(1-g b) R=(0)$ and $R(1-b g) a=(0)$. As $1 \in R$, $a(1-g b)=0$ and $(1-b g) a=0$. Therefore, $b g a=a=a g b$. Now for any $t_{1}, t_{2} \in R, a t_{1}=(b g a) t_{1}=b\left(g a t_{1}\right) \in b R$ and $(b-a) t_{2}=b t_{2}-a t_{2}=b t_{2}-(b g a) t_{2}=$ $b\left(t_{2}-g_{a t}\right) \in b R$. Hence, $a R+(b-a) R \subseteq b R$. Thus $a R+(b-a) R=b R$. Now we want to show that $a R \cap(b-a) R=(0)$. For some $u, v \in R$, suppose $a u=(b-a) v \in$ $a R \cap(b-a) R$. Then $a u=a g a u=a g(b-a) v=a g b v-a g a v=a v-a v=0$ as $a=a g b$. Thus $a R \cap(b-a) R=(0)$ and so $b R=a R \oplus(b-a) R$. Hence, $a \leq^{\oplus} b$ as required.

We also note that proving directly $(2) \Longrightarrow(1)$ requires a brief argument.

The Corollary that follows shows, in particular, that the minus partial order defined on the set of idempotents is the same as the partial order defined by Kaplansky on idempotents (See e.g. Lam [9], page 323). 
Corollary 4. Let $R$ be a regular ring and $a, b \in R$ such that $b=b^{2}$. Then the following are equivalent:

(1) $a \leq^{-} b$;

(2) $a=a^{2}=a b=b a$.

Proof. The proof is straightforward.

Corollary 5. Let $R$ be a regular ring and let $a, b, c \in R$ with $b=a+c$. Then the following statements are equivalent:

(1) $a \leq^{-} b$;

(2) $a R \cap c R=(0)=R a \cap R c$.

Proof. It follows from Lemma 3 and observing that, in a regular ring, $a \leq^{-} a+c$ if and only if $a \leq^{\oplus} a+c$ if and only if $(a+c) R=a R \oplus c R$.

Hartwig ([ $\underline{6}$, Pages 12-13) posed the following questions, among others:

(1) If $R$ is a regular ring and $a R \cap c R=(0)=R a \cap R c$, does there exist $a^{(1)}$ such that $a^{(1)} c=0=c a^{(1)}$ ?

(2) Does $a \leq^{-} c, b \leq^{-} c, a R \cap c R=(0)=R a \cap R c$ imply $a+b \leq^{-} c$ ?

As a byproduct of the development of the direct sum partial order, we give an application that answers the above two questions of Hartwig. We do not know whether or not someone has answered these questions, as we could not find this in the literature. In any case, we believe that the answers we have given would be of interest to the reader. Below, we answer Question 1 in the affirmative and Question 2 in the negative by providing a counterexample.

Proposition 6. (Hartwig Question 1) If $R$ is a regular ring and $a R \cap c R=(0)=$ $R a \cap R c$, for some nonzero elements $a, c \in R$, then there exists a nonzero $a^{(1)}$ such that $a^{(1)} c=0=c a^{(1)}$.

Proof. Let $b=a+c$. By Corollary $5, a \leq^{-} b$. Then, by the definition of the minus partial order, for some $a^{(1)}, a a^{(1)}=b a^{(1)}$ and $a^{(1)} a=a^{(1)} b$. Now substituting $b=a+c$ yields $a a^{(1)}=(a+c) a^{(1)}$ and $a^{(1)} a=a^{(1)}(a+c)$. Thus $a a^{(1)}=a a^{(1)}+c a^{(1)}$ and $a^{(1)} a=a^{(1)} a+a^{(1)} c$. It follows that $c a^{(1)}=0=a^{(1)} c$ as required.

Example 7. (Hartwig Question 2)

Using matrix units $e_{i j}$, let $a=e_{13}, b=e_{24}$, and $c=e_{13}+e_{14}+e_{24}$. Clearly $a \leq^{-} c$ and $b \leq^{-} c$. It is obvious that $a R \cap b R=(0)=R a \cap R b$. Since rank $(c)-$ $\operatorname{rank}(a+b)=2-2=0$ and $\operatorname{rank}(c-(a+b))=1$, it follows that $a+b \not^{-} c$. 


\section{Main Results}

Let $R$ be a regular ring and $S$ be a subset of $R$. We define a maximal element in $C=\left\{x \in S: x \leq^{\oplus} a\right\}$ as an element $b \neq a$ such that $b \leq^{\oplus} a$ and if $b \leq^{\oplus} c \leq^{\oplus} a$ then $c=b$ or $c=a$.

For fixed elements $a, b, c \in R$, we give a complete description of the maximal elements in the subring $S=e R f$, where $e$ and $f$ are idempotents given by $e R=$ $a R \cap c R$ and $R f=R a \cap R b$. Here, $C=\left\{s \in e R f: s \leq^{\oplus} a\right\}$. In the literature, maximal elements in $C$ have been called shorted operators of $a$ ([1, 2] and [13).

We begin with a result that is used frequently in the sequel. This is indeed contained in (15], Lemma 1) where the author proves the equivalence of 11 statements. However, for the sake of completeness, we provide a direct argument.

Lemma 8. Suppose $R$ is a regular ring and $a, b \in R$ such that $\left\{a^{(1)}\right\} \cap\left\{b^{(1)}\right\} \neq \emptyset$. Then the following are equivalent:

(1) $a R \subset b R$ and $R a \subset R b$;

(2) $a \leq^{\oplus} b$.

Proof. Suppose $a R \subset b R$ and $R a \subset R b$. It follows that $a=r b=b s$ for some $r, s \in$ $R$. We claim that $a b^{(1)} a$ is invariant under any choice of $b^{(1)}$. Let $x, y \in\left\{b^{(1)}\right\}$ be arbitrary. Now $a x a=(r b) x(b s)=r(b x b) s=r b s$ as $b x b=b$. Similarly, aya $=$ $(r b) y(b s)=r(b y b) s=r b s$ as $b y b=b . \quad$ Thus $a x a=a y a$ for every $x, y \in\left\{b^{(1)}\right\}$. Hence $a b^{(1)} a$ is invariant under any choice of $b^{(1)}$. Since we have assumed that $\left\{a^{(1)}\right\} \cap\left\{b^{(1)}\right\} \neq \emptyset$, there exists some $g \in\left\{a^{(1)}\right\} \cap\left\{b^{(1)}\right\}$. Therefore $a b^{(1)} a=a g a=a$ for all $b^{(1)}$. Hence $\left\{b^{(1)}\right\} \subseteq\left\{a^{(1)}\right\}$ and by Lemma 3, $a \leq^{\oplus} b$.

Conversely, if $a \leq^{\oplus} b$, then $a R \subset b R$ and $R a \subset R b$ follow by definition.

We now demonstrate an important relationship between weak von Neumann inverses and strong von Neumann inverses under the direct sum partial order.

Lemma 9. Let $a \in R$ where $R$ is a regular ring. Then the following are equivalent:

(1) $b$ is a weak von Neumann inverse of $a$;

(2) There exists a strong von Neumann inverse $c$ of a such that $b \leq^{\oplus} c$.

Proof. Suppose $b$ is a weak von Neumann inverse of $a$. For any fixed $a^{(1)}$, define $u=a^{(1)}(a-a b a) a^{(1)}$ and $c=b+u$. Then $a c a=a b a+a u a=a b a+a a^{(1)} a a^{(1)} a-$ $a a^{(1)} a b a a^{(1)} a=a b a+a-a b a=a$ and $c a c=(b+u) a(b+u)=b a b+b a u+u a b+u a u=$ $b+b a\left(a^{(1)} a a^{(1)}-a^{(1)} a b a a^{(1)}\right)+\left(a^{(1)} a a^{(1)}-a^{(1)} a b a a^{(1)}\right) a b+$

$\left(a^{(1)} a a^{(1)}-a^{(1)} a b a a^{(1)}\right) a\left(a^{(1)} a a^{(1)}-a^{(1)} a b a a^{(1)}\right)=b+b a a^{(1)}-b a a^{(1)}+a^{(1)} a b-$ $a^{(1)} a b+a^{(1)} a a^{(1)}-a^{(1)} a b a a^{(1)}-a^{(1)} a b a a^{(1)}+a^{(1)} a b a a^{(1)}=b+a^{(1)}(a-a b a) a^{(1)}=$ $b+u=c$. This shows that $c$ is a strong von Neumann inverse of $a$.

Now we want to show that $b \leq^{\oplus} c$. In other words, we will prove that $b R \oplus u R=$ $c R$. Observe that $c a b=\left[b+a^{(\overline{1})}(a-a b a) a^{(1)}\right] a b=b a b+a^{(1)}(a b-a b a b)=b a b=b$. Therefore $b \in c R$. As $c=b+u$, it is clear that $c R \subseteq b R+u R$. As $u=c-b$ and $b \in c R, u R \subseteq c R$. It follows that $c R=b R+u R$. Now we want to show that $b R \cap u R=(0)$. Let $b p=u q \in b R \cap u R$ for some $p, q \in R$. Multiplying $b a$ on both sides yields $b p=b a b p=b a u q=b a\left[a^{(1)}(a-a b a) a^{(1)}\right] q=(b a-b a b a) a^{(1)} q=$ $(b a-b a) a^{(1)} q=0$. Therefore $b R \cap u R=0$. Thus $b R \oplus u R=c R$ and we have demonstrated that $b \leq^{\oplus} c$.

Conversely, suppose that there exists a strong von Neumann inverse $c$ of $a$ such that $b \leq^{\oplus} c$. As $c$ is a weak von Neumann inverse of $a, c a c=c$ and thus $a \in\left\{c^{(1)}\right\}$. 
By assumption $b \leq^{\oplus} c$ and it follows from Lemma 3 that $\left\{c^{(1)}\right\} \subseteq\left\{b^{(1)}\right\}$. Thus $a \in\left\{c^{(1)}\right\} \subseteq\left\{b^{(1)}\right\}$ and it follows that $b a b=b$. Hence $b$ is a weak von Neumann inverse of $a$.

Lemma 10. Suppose $R$ is a regular ring. Let y be a weak von Neumann inverse and $z$ be a strong von Neumann inverse of an element $\alpha$ in the subring $f$ Re such that $y \leq^{\oplus} z$. Then eyf $\leq^{\oplus}$ ezf.

Proof. Let $\alpha=f x e \in f R e$. Since $y \leq \oplus z, y R \subseteq z R$ and $R y \subseteq R z$. Thus, $y=r z=z s$ for some $r, s \in R$. It is straightforward to verify that $z \alpha y=y=y \alpha z$. This gives $(e z f) x(e y f)=(e z f) x(e(z s) f)=e z(f x e) z s f=e z s f=e y f$. Similarly $(e y f) x(e z f)=e y f$. Thus $(e y f) R \subseteq(e z f) R$ and $R(e y f) \subseteq R(e z f)$. As $\alpha=f x e$ is a common von Neumann inverse of $y$ and $z$, it follows that $(e y f) x(e y f)=e y f$ and $(e z f) x(e z f)=e z f$ and so $x$ is a common von Neumann inverse of eyf and $e z f$. By Lemma 8 , eyf $\leq^{\oplus}$ ezf .

Next, we give two key lemmas. We will assume throughout that $a \notin S$.

Lemma 11. Let $R$ be a regular ring. Then $d \in C$ is a maximal element in $C$ if and only if for any $d^{\prime} \leq^{\oplus}$ a such that $d R \subseteq d^{\prime} R \subseteq e R, R d \subseteq R d^{\prime} \subseteq R f$, we have $d=d^{\prime}$.

Proof. Let $d$ be a maximal element in $C$. If $d^{\prime}$ is any element in $R$ such that $d^{\prime} \leq^{\oplus} a$ and $d R \subseteq d^{\prime} R \subseteq e R, R d \subseteq R d^{\prime} \subseteq R f$, then clearly $d^{\prime} \in e R f$. As $d^{\prime} \leq^{\oplus} a$, $d^{\prime} \in C$. Then $\left\{\bar{a}^{(1)}\right\} \subseteq\left\{d^{(1)}\right\} \cap\left\{\left(d^{\prime}\right)^{(1)}\right\}$. Hence, $d \leq^{\oplus} d^{\prime}$ by Lemma 8 . Then by the maximality of $d$ in $C, d=d^{\prime}$.

The converse is obvious.

Lemma 12. $C=\left\{\right.$ euf $: u$ is a weak von Neumann inverse of $\left.f a^{(1)} e\right\}$.

Proof. Let $s=e t f \in C$ for some $t \in R$. Then $s \leq^{\oplus} a$. By Lemma 3, $\left\{a^{(1)}\right\} \subseteq$ $\left\{s^{(1)}\right\}$. Therefore, we have $($ etf $) a^{(1)}($ etf $)=($ et $f)$. In other words, $($ et $f)\left(f a^{(1)} e\right)($ etf $)=$ (etf), proving that $s=e t f$ is a weak von Neumann inverse of $f a^{(1)} e$. This shows that $s=e u f$ for some weak von Neumann inverse $u$ of $f a^{(1)} e$.

Conversely, consider any $u \in\left(f a^{(1)} e\right)^{(2)}$ and let $x=e u f$. We want to show that $x \leq^{\oplus} a$. Now $x a^{(1)} x=($ euf $) a^{(1)}(e u f)=e u\left(f a^{(1)} e\right) u f=e u f=x$ as $u \in\left(f a^{(1)} e\right)^{(2)}$. Hence $\left\{a^{(1)}\right\} \subseteq\left\{x^{(1)}\right\}$. By Lemma 3, $x \leq^{\oplus} a$ and so $x=e u f \in C$.

Theorem 13. $\max C=\left\{e v f: v\right.$ is a strong von Neumann inverse of $\left.f a^{(1)} e\right\}$.

Proof. Suppose $x=$ euf $\in C$ where $u=\left(f a^{(1)} e\right)^{(2)}$. By Lemma 9, there is a strong von Neumann inverse $v \in e R f$ of $f a^{(1)} e$ such that $u \leq^{\oplus} v$ and consequently, by Lemma 10, euf $\leq^{\oplus}$ evf. Thus, we have $x \leq^{\oplus}$ evf. Next, we will show that $e v f \leq^{\oplus} a$. We have $(e v f) a^{(1)}(e v f)=e v\left(f a^{(1)} e\right) v f=e v f$ as $v \in\left(f a^{(1)} e\right)^{(1,2)}$. Hence $\left\{a^{(1)}\right\} \subseteq\left\{(e v f)^{(1)}\right\}$. By Lemma 3, evf $\leq^{\oplus} a$. Thus max $C \subseteq\{e v f: v$ is a strong von Neumann inverse of $\left.f a^{(1)} e\right\}$. Clearly, $\max C$ is non-empty unless $e v f=a$ for each choice of $v$ but this is not possible as we have assumed $a \notin S$.

Now suppose $e v f, e v^{\prime} f \in C$ such that $v, v^{\prime}$ are strong von Neumann inverses of $f a^{(1)} e$ and $e v f \leq{ }^{\oplus} e v^{\prime} f$. Therefore $e v^{\prime} f R=e v f R \oplus\left(e v^{\prime} f-e v f\right) R$. Now we want to show that $e v^{\prime} f R=e v f R$. As $e v f, e v^{\prime} f \in C, e v f \leq^{\oplus} a$ and $e v^{\prime} f \leq^{\oplus} a$. Thus $\left\{a^{(1)}\right\} \subseteq\left\{(e v f)^{(1)}\right\}$ and $\left\{a^{(1)}\right\} \subseteq\left\{\left(e v^{\prime} f\right)^{(1)}\right\}$. So let $a^{(1)}$ be a common von 
Neumann inverse of $e v f$ and $e v^{\prime} f$. By assumption $e v f R \subseteq e v^{\prime} f R$. As shown in Lemma 10, $\left(e v^{\prime} f\right) a^{(1)}(e v f)=e v f$ and $\left(e v^{\prime} f\right) a^{(1)}\left(e v^{\prime} f\right)=\left(e v^{\prime} f\right)$. Now $\left(e v^{\prime} f\right) R=$ $e v^{\prime} f a^{(1)} R=e v^{\prime} f a^{(1)} e R=e v^{\prime}\left(f a^{(1)} e v f a^{(1)} e\right) R \subseteq e v^{\prime} f a^{(1)} e v f R=e v f R \subseteq e v^{\prime} f R$. Thus $e v^{\prime} f R=e v f R$. Similarly we can show that $R e v^{\prime} f=R e v f$.

As $\operatorname{Rev}^{\prime} f=\operatorname{Revf}$, we claim that $e v^{\prime} f=e v f$. Let $e v^{\prime} f=r e v f$ for some $r \in R$. Now $e v f=e v^{\prime} f a^{(1)} e v f=(r e v f) a^{(1)} e v f=r(e v f)=e v^{\prime} f$. Thus $e v f=e v^{\prime} f$. Hence $\max C=\left\{e v f: v\right.$ is a strong von Neumann inverse of $\left.f a^{(1)} e\right\}$.

We now provide an example to illustrate the previous theorem.

Example 14. Note that we are choosing $f$ to be of rank two. So any maximal element will have, at most, rank two. Choose $e=\left[\begin{array}{cccc}1 & 0 & 0 & 0 \\ 0 & \frac{1}{2} & \frac{1}{2} & 0 \\ 0 & \frac{1}{2} & \frac{1}{2} & 0 \\ 0 & 0 & 0 & 1\end{array}\right]$ and $f=\left[\begin{array}{cccc}\frac{1}{2} & \frac{1}{4} & 0 & 0 \\ 1 & \frac{1}{2} & 0 & 0 \\ 0 & 0 & 0 & 0 \\ 0 & 0 & 0 & 1\end{array}\right]$. Suppose $a=\left[\begin{array}{cccc}1 & 0 & 0 & 0 \\ 0 & 1 & 0 & 0 \\ 0 & 0 & 1 & 0 \\ 0 & 0 & 0 & 1\end{array}\right]$. Then one choice for $a^{(1)}$ is $a^{(1)}=\left[\begin{array}{llll}1 & 0 & 0 & 0 \\ 0 & 1 & 0 & 0 \\ 0 & 0 & 1 & 0 \\ 0 & 0 & 0 & 1\end{array}\right]$ and $f a^{(1)} e=\left[\begin{array}{cccc}\frac{1}{2} & \frac{1}{8} & \frac{1}{8} & 0 \\ 1 & \frac{1}{4} & \frac{1}{4} & 0 \\ 0 & 0 & 0 & 0 \\ 0 & 0 & 0 & 1\end{array}\right]$. For our choice of $a$ strong von Neumann inverse of $f a^{(1)} e$, we first choose its Moore-Penrose inverse and later its group inverse, as both are also strong von Neumann inverses. Let $v_{1}$ be the Moore-Penrose inverse of $\mathrm{fa}^{(1)}$ e. Then $v_{1}=\left[\begin{array}{cccc}\frac{16}{45} & \frac{32}{45} & 0 & 0 \\ \frac{4}{45} & \frac{8}{45} & 0 & 0 \\ \frac{4}{45} & \frac{8}{45} & 0 & 0 \\ 0 & 0 & 0 & 1\end{array}\right]$ and $e v_{1} f=\left[\begin{array}{cccc}\frac{8}{9} & \frac{4}{9} & 0 & 0 \\ \frac{2}{9} & \frac{1}{9} & 0 & 0 \\ \frac{2}{9} & \frac{1}{9} & 0 & 0 \\ 0 & 0 & 0 & 1\end{array}\right] . \quad$ Now ev $v_{1} f \leq^{-}$a because $\operatorname{rank}\left(a-e v_{1} f\right)=2=4-2=$ $\operatorname{rank}(a)-\operatorname{rank}\left(e v_{1} f\right)$. Thus $e v_{1} f \in \max C$.

We now find another element of $\max C$. The group-inverse $v_{2}$ of $f a^{(1)} e$ is $v_{2}=\left[\begin{array}{cccc}\frac{8}{9} & \frac{2}{9} & \frac{2}{9} & 0 \\ \frac{16}{9} & \frac{4}{9} & \frac{4}{9} & 0 \\ 0 & 0 & 0 & 0 \\ 0 & 0 & 0 & 1\end{array}\right]$. Then ev $f=\left[\begin{array}{cccc}\frac{2}{3} & \frac{1}{3} & 0 & 0 \\ \frac{2}{3} & \frac{1}{3} & 0 & 0 \\ \frac{2}{3} & \frac{1}{3} & 0 & 0 \\ 0 & 0 & 0 & 1\end{array}\right]$. Now ev $f \leq^{-}$a because $\operatorname{rank}\left(a-e v_{2} f\right)=2=4-2=\operatorname{rank}(a)-\operatorname{rank}\left(e v_{2} f\right) . \quad T h u s e v_{2} f \in \max C$.

\section{An Applichtion}

In this section, as an application of our main theorem on maximal elements, we derive the unique shorted operator $a_{S}$ of Anderson-Trapp (See [2, Theorem 1) that was also studied by Mitra-Puri (See [13, Theorem 2.1). We believe that there will be other such applications. 
Throughout this section $R$ will denote the ring of $n \times n$ matrices over the field of complex numbers, $\mathbb{C}$. For any matrix or vector $u, u^{*}$ will denote the conjugate transpose of $u$. In this section $S$ will denote the set of positive semidefinite matrices.

Recall, the Loewner order, $\leq_{L}$, on the set $S$ of positive semidefinite matrices in $R$ is defined as follows: for $a, b \in S, a \leq_{L} b$ if $b-a \in S$.

Suppose $a \in S$ and $c \in R$. As in the previous section, $e R=a R \cap c R, e=e^{2}$, and choose $f=e^{*}$. Clearly, $f \in R a$ because $a$ is hermitian. Let $C_{L}=\{s \in$ $\left.e R f \cap S: s \leq_{L} a\right\}=\left\{s \in e S f: s \leq_{L} a\right\}$.

Under this terminology, the set $C$ in the previous section will become, $C=\{s \in$ $e S f: s \leq \oplus a\}$.

We will assume that $a \notin e S f$. This is equivalent to the assumption that $\operatorname{rank}(e) \neq \operatorname{rank}(a)$, as shown in the remark below.

Remark 15. $\operatorname{rank}(e)=\operatorname{rank}(a)$ if and only if $a \in e S f$.

Proof. Suppose $\operatorname{rank}(e)=\operatorname{rank}(a)$. So $e R=a R$ as $e R \subseteq a R$. Then $a=e x$ for some $x \in R$ and by taking conjugates, $a=x^{*} e^{*}$, i.e., $a \in R e^{*}$. Hence, $a \in e R e^{*}$. As $a \in S, a \in S \cap e R e^{*}=e S e^{*}$. For if $e x e^{*} \in S$ then $e x e^{*}=e\left(e x e^{*}\right) e^{*} \in e S e^{*}$ and so $S \cap e R e^{*} \subseteq e S e^{*}$. The reverse inclusion is obvious.

Conversely, suppose $a \in e S f$. As $e R=a R \cap c R$, we have $e=a x$ and so $\operatorname{rank}(e) \leq \operatorname{rank}(a)$. As $a \in e S f, a=e s e^{*}$ for some $s \in S$. Therefore $\operatorname{rank}(a) \leq$ $\operatorname{rank}(e)$. Hence, $\operatorname{rank}(e)=\operatorname{rank}(a)$.

The following lemma is folklore.

Lemma 16. Suppose $a, b \in S$. If $a \leq^{\oplus} b$ then $a \leq_{L} b$.

Proof. Suppose $a \leq^{\oplus} b$. Equivalently, $(b-a) \leq^{\oplus} b$ and by Lemma 3 we know that $\left\{b^{(1)}\right\} \subseteq\left\{(b-a)^{(1)}\right\}$. Thus, $b^{\dagger}$ is a von Neumann inverse of $(b-a)$. From [11], as $b$ is positive semidefinite, $b^{\dagger}$ is positive semidefinite. Thus $b-a=$ $(b-a) b^{\dagger}(b-a) \geq_{L} 0$. Hence $(b-a) \in S$ and $a \leq_{L} b$.

Theorem 17. Let $a \in S$ and let $f_{a}^{\dagger}$ be the a-weighted Moore-Penrose inverse of f. Then $\max C=\max C_{L}=\left\{a f_{a}^{\dagger} f\right\}$.

Proof. By Theorem 13, $\max C=\{e v f: v$ is a strong von Neumann inverse of $\left.f a^{(1)} e\right\}$. By assumption, $e \in a R$ and so $e=a x$ for some $x \in R$. By taking conjugates, $e^{*}=x^{*} a$ as $a \in S$. In addition, as $f \in R a, f=y a$ for some $y \in R$. This yields that $f a^{(1)} e=y a a^{(1)} a x=y a x$ and thus $f a^{(1)} e$ is independent of the choice of $a^{(1)}$. We may then choose the Moore-Penrose inverse $a^{\dagger}$ for $a^{(1)}$. Next, we want to show that a strong von Neumann inverse of $f a^{\dagger} e$ is also unique. Note that $f a^{\dagger} e=e^{*} a^{\dagger} e$ is positive semidefinite, as the Moore-Penrose inverse of a positive semidefinite element is positive semidefinite [11]. As $a \in S$, we can write $a=z z^{*}$ for some $z \in R$. Now $f R=y a R=y a a^{\dagger} a R=f a^{\dagger} a R=f a^{\dagger} R=f z z^{*} R=$ $f z R=(f z)(f z)^{*} R=f z z^{*} f^{*} R=f a^{\dagger} e R$. Similarly $R e=R f a^{\dagger} e$. It follows that $f=f a^{\dagger} e p$ and $e=q f a^{\dagger} e$ for some $p, q \in R$. Consider an element $e v f \in$ $\max C$. Then $e v f=q f a^{\dagger} e v f a^{\dagger} e p=q f a^{\dagger} e p$, showing that $e v f$ is independent of the choice of strong von Neumann inverse $v$ of $f a^{\dagger} e$. Thus $\max C$ is a singleton set consisting of the element $e\left(f a^{\dagger} e\right)^{\dagger} f$. Since $a \in S, a^{\dagger} \in S$ and hence $e\left(e^{*} a^{\dagger} e\right)^{\dagger} f=$ $e\left(f a^{\dagger} e\right)^{\dagger} f \in S$. 
Next, we proceed to show that $\max C=\left\{a f_{a}^{\dagger} f\right\}$ also. Recall that $a f_{a}^{\dagger} f$ is hermitian and so $a f_{a}^{\dagger} f=\left(a f_{a}^{\dagger} f\right)^{*}=f^{*}\left(f_{a}^{\dagger}\right)^{*} a^{*}=\left(f_{a}^{\dagger} f\right)^{*} a$. Since $f_{a}^{\dagger} f$ is an idempotent, we get $a f_{a}^{\dagger} f=a\left(f_{a}^{\dagger} f\right)\left(f_{a}^{\dagger} f\right)=\left(f_{a}^{\dagger} f\right)^{*} a\left(f_{a}^{\dagger} f\right)$ and thus $a f_{a}^{\dagger} f \in S$.

We now prove that $a f_{a}^{\dagger} f \leq^{\oplus} a$. Let $a^{(1)}$ be an arbitrary von Neumann inverse of $a$. Then $\left(a f_{a}^{\dagger} f\right) a^{(1)}\left(a f_{a}^{\dagger} f\right)=\left(a f_{a}^{\dagger}\right)(y a) a^{(1)}\left(a f_{a}^{\dagger} f\right)=\left(a f_{a}^{\dagger} y\right) a a^{(1)} a\left(f_{a}^{\dagger} f\right)=$ $a f_{a}^{\dagger} y a f_{a}^{\dagger} f=a f_{a}^{\dagger} f f_{a}^{\dagger} f=a f_{a}^{\dagger} f$. Hence $\left\{a^{(1)}\right\} \subseteq\left\{\left(a f_{a}^{\dagger} f\right)^{(1)}\right\}$. Consequently, by Lemma $3, a f_{a}^{\dagger} f \leq \oplus a$ which gives $a f_{a}^{\dagger} f \in C$.

Furthermore, by Lemma 16, $a f_{a}^{\dagger} f \leq^{\oplus} a$ gives $a f_{a}^{\dagger} f \leq_{L} a$ and hence $a f_{a}^{\dagger} f \in C_{L}$.

Finally, we show that for every $d \in \bar{C}_{L}, d \leq_{L} a f_{a}^{\dagger} f$. As $d \in S \subseteq R f$, write $d=u f$ for some $u \in R$. Then $d f_{a}^{\dagger} f=u f f_{a}^{\dagger} f=u f=d=\left(f_{a}^{\dagger} f\right)^{*} d\left(f_{a}^{\dagger} f\right)$ as $d$ is hermitian. Now consider $a f_{a}^{\dagger} f-d=\left(f_{a}^{\dagger} f\right)^{*} a\left(f_{a}^{\dagger} f\right)-\left(f_{a}^{\dagger} f\right)^{*} d\left(f_{a}^{\dagger} f\right)=\left(f_{a}^{\dagger} f\right)^{*}(a-d)\left(f_{a}^{\dagger} f\right)$, which is positive semidefinite and thus $a f_{a}^{\dagger} f-d \in S$. Hence $d \leq_{L} a f_{a}^{\dagger} f$.

Thus $a f_{a}^{\dagger} f$ is the unique maximal element in $C_{L}$ provided $a f_{a}^{\dagger} f \neq a$. We have shown above that $a f_{a}^{\dagger} f \in C_{L}$ and thus $a f_{a}^{\dagger} f \in e S f$. But by assumption $a \notin e S f$. So $a f_{a}^{\dagger} f \neq a$. Therefore, $a f_{a}^{\dagger} f$ is unique maximal element in $C_{L}$ and it also belongs to $C$ as we have already proven that $a f_{a}^{\dagger} f \leq^{\oplus} a$.

Now, because $e\left(f a^{\dagger} e\right)^{\dagger} f$ is the unique maximal element in $C$ and $a f_{a}^{\dagger} f \in C$, $a f_{a}^{\dagger} f \leq^{\oplus} e\left(f a^{\dagger} e\right)^{\dagger} f$. By Lemma 16, $a f_{a}^{\dagger} f \leq_{L} e\left(f a^{\dagger} e\right)^{\dagger} f$ as $e\left(f a^{\dagger} e\right)^{\dagger} f \in C_{L}$. We have shown above that for every element $d \in C_{L}, d \leq_{L} a f_{a}^{\dagger} f$ and thus $a f_{a}^{\dagger} f=$ $e\left(f a^{\dagger} e\right)^{\dagger} f$. Hence, $\max C=\max C_{L}=\left\{a f_{a}^{\dagger} f\right\}$ as desired.

The following examples demonstrate the result proved in the previous theorem, i.e. $a f_{a}^{\dagger} f=e\left(f a^{\dagger} e\right)^{\dagger} f$ and so $\max C=\max C_{L}=\left\{a f_{a}^{\dagger} f\right\}$. Furthermore, $\max C$ agrees with the formula given by Anderson-Trapp for computing the shorted operator $a_{S}$ when we are given the impedance matrix $a$.

The Anderson-Trapp formula states that if $a$ is the $n \times n$ impedance matrix then the shorted operator of $a$ with respect to the $k$-dimensional subspace $S$ (shorting $n-k$ ports) is given by $a_{S}=\left[\begin{array}{cc}a_{11}-a_{12} a_{22}^{\dagger} a_{21} & 0 \\ 0 & 0\end{array}\right]$, where $a$ is partitioned as $a=\left[\begin{array}{ll}a_{11} & a_{12} \\ a_{21} & a_{22}\end{array}\right]$ such that $a_{11}$ is a $k \times k$ matrix. We show that the maximum element $a f_{a}^{\dagger} f$ obtained by us is permutation equivalent to $a_{S}$, i.e. $P^{T} a f_{a}^{\dagger} f P=a_{S}$ for some permutation matrix $P$.

Example 18. Let $e=\left[\begin{array}{cccc}\frac{1}{2} & 0 & \frac{1}{2} & 0 \\ 0 & 0 & 0 & 0 \\ \frac{1}{2} & 0 & \frac{1}{2} & 0 \\ 0 & 0 & 0 & 1\end{array}\right]$ and then $f=e^{*}=\left[\begin{array}{cccc}\frac{1}{2} & 0 & \frac{1}{2} & 0 \\ 0 & 0 & 0 & 0 \\ \frac{1}{2} & 0 & \frac{1}{2} & 0 \\ 0 & 0 & 0 & 1\end{array}\right]$. Suppose

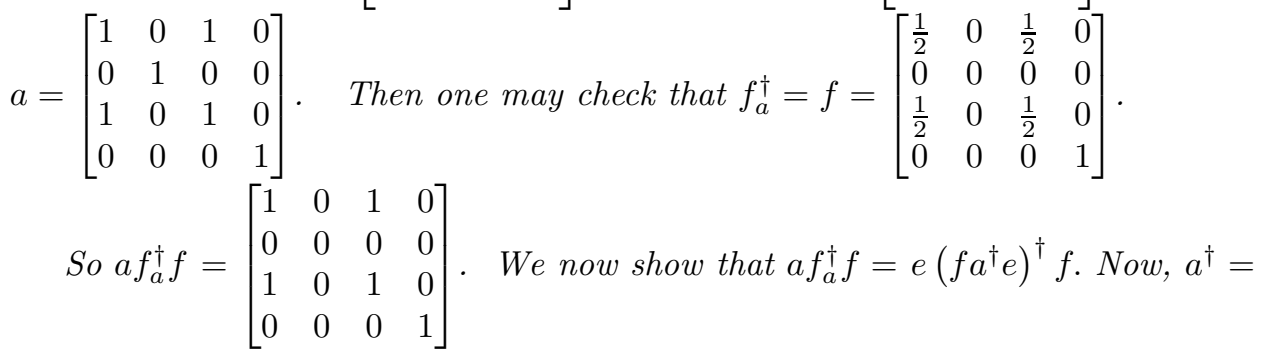


$\left[\begin{array}{cccc}\frac{1}{4} & 0 & \frac{1}{4} & 0 \\ 0 & 1 & 0 & 0 \\ \frac{1}{4} & 0 & \frac{1}{4} & 0 \\ 0 & 0 & 0 & 1\end{array}\right]$ and $\left(f a^{\dagger} e\right)^{\dagger}=\left[\begin{array}{cccc}1 & 0 & 1 & 0 \\ 0 & 0 & 0 & 0 \\ 1 & 0 & 1 & 0 \\ 0 & 0 & 0 & 1\end{array}\right]$. Thus e $\left(f a^{\dagger} e\right)^{\dagger} f=\left[\begin{array}{cccc}1 & 0 & 1 & 0 \\ 0 & 0 & 0 & 0 \\ 1 & 0 & 1 & 0 \\ 0 & 0 & 0 & 1\end{array}\right]$.

Hence $e\left(f a^{\dagger} e\right)^{\dagger} f=\left[\begin{array}{llll}1 & 0 & 1 & 0 \\ 0 & 0 & 0 & 0 \\ 1 & 0 & 1 & 0 \\ 0 & 0 & 0 & 1\end{array}\right]=a f_{a}^{\dagger} f$ as proved in the theorem. We may verify that $a f_{a}^{\dagger} f \leq^{\oplus} a$. This follows from $\operatorname{rank}(a)-\operatorname{rank}\left(a f_{a}^{\dagger} f\right)=3-2=1=$ $\operatorname{rank}\left(a-a f_{a}^{\dagger} f\right)$. We know then $a f_{a}^{\dagger} f \leq_{L} a$. Thus $\max C=\max C_{L}=\left\{a f_{a}^{\dagger} f\right\}$.

We now compute the shorted operator as given by Anderson-Trapp. We partition a as follows: $a=\left[\begin{array}{lll}{\left[\begin{array}{lll}1 & 0 & 1 \\ 0 & 1 & 0 \\ 1 & 0 & 1\end{array}\right]} & {\left[\begin{array}{l}0 \\ 0 \\ 0\end{array}\right]} \\ 0 & 0 & 0\end{array}\right]$.

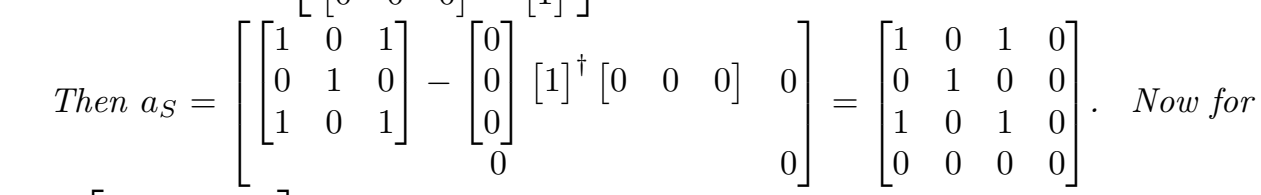
$P=\left[\begin{array}{llll}1 & 0 & 0 & 0 \\ 0 & 0 & 0 & 1 \\ 0 & 0 & 1 & 0 \\ 0 & 1 & 0 & 0\end{array}\right], P a f_{a}^{\dagger} f P^{T}=a_{S}$

\section{REFERENCES}

[1] W. N. Anderson Jr., Shorted operators, SIAM J. Appl. Math. 20 (1971), 522-525.

[2] W. N. Anderson Jr., G. E. Trapp, Shorted operators II, SIAM J. Appl. Math. 28 (1975), 60-71.

[3] A. Ben-Israel, T. N. E. Greville, Generalized Inverses: Theory and Applications, second ed., Springer-Verlag, Berlin, 2002.

[4] M. P. Drazin, Natural Structures On semigroups with involution, Bull. Amer. Math. Soc. 84 (1978), 139-141.

[5] K. R. Goodearl, von Neumann Regular Rings, second ed., Krieger Publishing Company, Malabar, Florida, 1991.

[6] R. E. Hartwig, How to partially order regular elements, Math. Japon. 25 (1980) 1-13.

[7] S. K. Jain, S. K. Mitra and H. J. Werner, Extensions of $G$-based matrix partial orders, SIAM J. Matrix Anal. Appl. 17 (1996), 834-850.

[8] S. K. Jain and K. M. Prasad, Right-Left symmetry of $a R \oplus b R=(a+b) R$ in Regular Rings, J. Pure and Applied Algebra, 133 (1998) 141-142.

[9] T. Y. Lam, A First Course in Noncommutative Rings, second ed., Springer-Verlag, 2001.

[10] T. Y. Lam, Lectures on Modules and Rings, Graduate Texts in Mathematics, Springer, 1998.

[11] T. O. Lewis and T. G. Newman, Pseudoinverses of positive semidefinite matrices, SIAM J. Appl. Math., 16 (1968) 701-703.

[12] K. Loewner, Über monotone matrixfunktionen, Math. Zeitschrift 38 (1934), 177-216.

[13] S. K. Mitra and Puri, M. L., Shorted operators and generalized inverses of matrices, Linear Algebra Appl. 25 (1979), 45-56.

[14] S. K. Mitra, The minus partial order and shorted matrix, Linear Algebra Appl. 83 (1986), 1-27.

[15] H. A. Mitsch, A natural partial order for semigroups, Proc. Amer. Soc. 97 (3) (1986), 384-388.

[16] K. S. S. Nambooripad, The natural partial order on a regular semigroup, Proc. Edinburgh Math. Soc. 23 (1980) 249-260.

[17] C. R. Rao, S. K. Mitra, Generalized Inverse of Matrices and Its Applications, first ed., Wiley, New York, 1971. 
Department of Mathematics, Potomac State College, West Virginia University, Keyser, West Virginia, USA

E-mail address: Brian.Blackwood@mail.wvu.edu

Department of Mathematics, Ohio University, Athens, Ohio-45701, USA

E-mail address: jain@math.ohiou.edu

Manipal Institue of Management, Manipal University, Manipal 576 104, Karnataka, INDIA

E-mail address: karantha.prasad@gmail.com

Department of Mathematics and Computer Science, St. Louis University, St. Louis, MO-63103, USA

E-mail address: asrivas3@slu.edu 